Jennifer Menon, Louise Maranda and Benjamin U. Nwosu*

\title{
Serum 25-hydroxyvitamin D levels do not correlate with asthma severity in a case-controlled study of children and adolescents
}

\section{Abstract}

Background: There is no consensus on the association between vitamin $\mathrm{D}$ and asthma.

objective: To determine the relationship between 25-hydroxyvitamin D [25(OH)D] levels and asthma symptom severity in children and adolescents.

Methods: A retrospective, case-control study of 263 subjects of ages 2-19 years with asthma who were compared to 284 non-asthmatic controls of similar ages. Subjects were excluded if they had diseases of calcium or vitamin D metabolism or were receiving calcium or vitamin D supplementation. Serum 25(OH)D was measured in all subjects. Asthma symptom severity, usually stratified into 6 steps, was stratified into five steps [1-5] based on the number and dose of controller medications used as outlined by the National Heart, Lung, and Blood Institute's guidelines. Mean 25(OH)D values were compared between the asthmatic patients and controls, as well as among the five steps of asthma symptom severity. Results were adjusted for age, sex, BMI, race and severity of asthma symptoms.

Results: There was no difference in $25(\mathrm{OH}) \mathrm{D}$ between asthmatic patients and controls $(28.64 \pm 10.09$ vs. $28.42 \pm 11.47$, $\mathrm{p}=1.0$ ). However, there was a significant difference in 25(OH)D between obese and non-obese asthmatic patients ( $23.33 \pm 7.67$ vs. $30.16 \pm 10.20, p<0.0001)$, as well as obese and non-obese controls ( $24.56 \pm 9.90$ vs. $29.50 \pm 11.66$, $\mathrm{p}=0.003)$. Mean $25(\mathrm{OH}) \mathrm{D}$ levels did not vary significantly among the five steps of asthma symptom severity.

Conclusions: There were no differences in mean $25(\mathrm{OH})$ $\mathrm{D}$ levels between asthmatic patients and controls. Mean 25(OH)D level was significantly lower in both the obese asthmatic patients and obese controls. Asthma severity had no relationship to mean 25(OH)D levels.

Keywords: asthma; obesity; symptom severity; vitamin D.

\footnotetext{
*Corresponding author: Dr. Benjamin U. Nwosu, Assistant Professor, Division of Endocrinology, Department of Pediatrics, University of Massachusetts Medical School, 55 Lake Avenue North, Worcester, MA 01655, USA,
}

Phone: +508-334-7872, Fax: +508-856-4287,

E-mail: Benjamin.Nwosu@umassmemorial.org Jennifer Menon: Department of Pediatrics, University of Massachusetts Medical School, Worcester, MA, USA Louise Maranda: Department of Pediatrics, University of Massachusetts Medical School, Worcester, MA, USA

\section{Introduction}

Asthma is the most common chronic condition in children and adolescents in the USA. There is no consensus on the association between asthma and vitamin $\mathrm{D}$. This immunemediated disease is characterized by reversible small airway inflammation leading to episodes of wheezing and respiratory distress. Despite the well-established National Asthma Education and Prevention Program set up by the National Heart, Lung, and Blood Institute (NHLBI) for the care and management of children and adolescents with asthma (1), the prevalence of the disease has continued to rise (2). There is a renewed interest in developing an adjunctive therapy to reduce the prevalence and severity of asthma.

This interest has led to the investigation of the immuno-modulatory properties of vitamin $\mathrm{D}$ as an antiinflammatory agent for the management of asthma (3). This is strengthened by epidemiological studies showing similarities in risk factors for both vitamin D deficiency and asthma. These similarities include African-American ethnicity, inner-city residence, obesity (4) and an increased asthma prevalence in the northern geographical latitudes in the USA, where exposure to sunlight is limited (5). These epidemiological and demographic relationships between asthma and vitamin D have led some investigators to hypothesize that as populations grow more prosperous and more Westernized, more time is spent indoors with less exposure to sunlight, leading to vitamin D deficiency, resulting in more asthma and allergy (6).

Vitamin D receptors (VDRs) have been identified on cells of the extra-skeletal system, especially those of the immune system, including human leukocytes, activated T cells and monocytes $(7,8)$. VDRs have also been identified 
on respiratory epithelium and bronchial smooth muscle cells. It has been postulated that vitamin D could enhance airway remodeling after an asthma exacerbation (3, 9-11). Vitamin D possesses anti-inflammatory properties (3) and enhances glucocorticoid response in steroid-resistance in asthma $(3,12)$.

Several studies have examined the beneficial effects of vitamin D on asthma symptom severity as well as its use as a surrogate marker of disease severity. In one large cohort study in asthmatic children in Costa Rica, Brehm et al. (13) reported an inverse correlation between serum concentrations of 25-hydroxyvitamin D [25(OH)D] levels and markers of allergy and asthma severity. Another study from northern Italy showed a direct correlation between asthma control and serum 25(OH)D in a cohort of asthmatic children (14). Other cross-sectional studies in children have shown that serum 25(OH)D concentrations are directly correlated with pulmonary function parameters, such as forced expiratory volume in one second (15), and also inversely correlated with asthma severity and airway smooth muscle mass (16).

Goleva et al. (17) reported a significant inverse correlation between serum IgE and serum vitamin D levels in pediatric patients with asthma but not in adult asthmatic patients. They also found significant associations between serum vitamin D levels and vitamin D-regulated targets and the patients' steroid responses in vitro, as well as steroid requirements in pediatric asthmatic patients but not in the adult asthmatic patients. These findings suggest that children with asthma might respond better to oral vitamin D supplementation than adults.

Some early effect studies have examined maternal 25(OH)D levels and the effects of vitamin D supplementation in early life on the development of asthma. Morales et al. (18) reported that higher maternal circulating $25(\mathrm{OH})$ D concentrations in pregnancy were independently associated with a lower risk of lower respiratory tract infections in offspring in the first year of life, but not with wheezing or asthma in childhood. However, another study from the northeastern US demonstrated that a higher maternal intake of vitamin D during pregnancy may decrease the risk of recurrent wheeze in early childhood (19).

In contrast, other studies have shown no evidence for low 25(OH)D levels in asthmatic subjects when compared to controls, whereas others have linked vitamin D sufficiency with adverse outcomes in asthma. A case-control study in two disparate adult populations in Europe did not find any significant difference in 25(OH)D levels between asthmatic patients and controls (20). Some studies have reported adverse associations between vitamin $\mathrm{D}$ and childhood eczema and asthma (21), and allergic diseases in adults $(22,23)$. Given all of the above differing conclusions, the Institute of Medicine recently reviewed the health effects of vitamin D and found no conclusive evidence for vitamin D supplementation in asthma and other extra-skeletal pathologies that have been associated with vitamin D deficiency (24).

We designed this retrospective, case-control study to further explore the relationship between asthma and vitamin D levels in children and adolescents. Our aims were to (A) determine if there was a significant difference in vitamin $D$ levels between asthmatic patients and controls, and (B) to determine if vitamin D levels were associated with asthma symptom severity. Our hypothesis was that 25(OH)D levels would be lower in patients with asthma.

\section{Methods}

\section{Subjects}

We reviewed the medical records of children and adolescents of ages 2-19 years with a diagnosis of asthma at the Children's Medical Center at the University of Massachusetts Medical Center between 2007 and 2011. The study protocol was approved by the University of Massachusetts Institutional Review Board. Study subjects ( $n=263$; 119 males) were included if they had a diagnosis of asthma or exercise-induced asthma and if they had a measured serum 25(OH)D level available. Patients were excluded if they had a diagnosis of any chronic lung disease other than the aforementioned diseases, such as cystic fibrosis, chronic lung disease of prematurity, bronchopulmonary dysplasia or pulmonary fibrosis; a concurrent diagnosis of any disease that affects calcium or vitamin D metabolism, such as DiGeorge syndrome, pseudohypoparathyroidism, hypoparathyroidism, hyperparathyroidism, rickets, renal failure, renal tubular acidosis, hepatitis or infiltrative liver diseases, history of vitamin D or calcium supplementation prior to the date of $25(\mathrm{OH}) \mathrm{D}$ measurement or were on continuous doses of oral corticosteroids for the control of any disease other than asthma. We further excluded pregnant or lactating females and patients who had 25(OH)D levels drawn during the course of an acute asthma exacerbation because patients undergoing asthma exacerbations do not meet the NHLBI's criteria for well-controlled asthma.

A group of age-matched children and adolescents served as controls. The controls were identified from the available medical records at the Children's Medical Center at the University of Massachusetts Medical Center between 2007 and 2011. Subjects were included in the control group ( $n=284 ; 117$ males) if they did not have a diagnosis of asthma, exercise-induced asthma, or reactive airway disease, but had serum 25(OH)D levels available. Control subjects were excluded if they had a diagnosis of any chronic lung disease, such as asthma, cystic fibrosis, chronic lung disease of prematurity, bronchopulmonary dysplasia, pulmonary hemosiderosis or pulmonary fibrosis, or diseases associated with calcium and vitamin D metabolism or if they were receiving calcium or vitamin D supplementation prior to the date of their serum 25(OH)D levels. We also excluded pregnant 
or lactating females and patients receiving systemic steroids. Ages of both study and control subjects was determined by the date of the 25(OH)D measurement. Subjects were categorized into non-obese and obese categories using a body mass index (BMI) of $>95^{\text {th }}$ percentile to define obesity (25).

\section{Anthropometry}

Height was measured to the nearest $0.01 \mathrm{~cm}$ using a wall-mounted stadiometer (Holtain Ltd, Crymych, Dyfed, UK). Weight was measured to the nearest $0.01 \mathrm{~kg}$ using an upright scale. BMI was derived using the formula weight $/$ height ${ }^{2}\left(\mathrm{~kg} / \mathrm{m}^{2}\right)$. All values were expressed as a standard deviation score (SDS) for age and gender based on the National Center for Health Statistics data (26).

\section{Assays}

Serum levels of 25(OH)D were analyzed using 25-hydroxy chemiluminescent immunoassay (DiaSorin Liaison; Stillwater, MN), which has a $100 \%$ cross-reactivity with both vitamin $D_{2}$ (the plant derived form of vitamin $\mathrm{D}$ called ergocalciferol) and vitamin $\mathrm{D}_{3}$ (the animal-derived form known as cholecalciferol) and thus measures total serum $25(\mathrm{OH}) \mathrm{D}$ content. Its functional sensitivity is $4 \mathrm{ng} / \mathrm{mL}(10 \mathrm{nmol} / \mathrm{L})$, and its intra- and inter-assay coefficients of variation are $5 \%$ and $8.2 \%$, respectively.

\section{Vitamin D status}

Vitamin D status was defined using 25(OH)D values based on the criteria by the Pediatric Endocrine Society as follows: vitamin D deficiency $<15 \mathrm{ng} / \mathrm{mL}$ (37.5 nmol/L), insufficiency $15-20 \mathrm{ng} / \mathrm{mL}$ (37.5-50 $\mathrm{nmol} / \mathrm{L}$ ) and sufficiency $>20 \mathrm{ng} / \mathrm{mL}$ ( $>50 \mathrm{nmol} / \mathrm{L}$ ) (27).

\section{Asthma symptom severity}

Asthma symptom severity was determined based on the controller medications prescribed to the patients and recorded in their medical records as recommended by the NHLBI's Guidelines for the Diagnosis and Management of Asthma. These guidelines provide a means of stratifying asthma symptom severity based on the number and type of controller medications and the dosages required to keep an asthmatic patient in a well-controlled state (1). There are six steps [1-6] that represent increasing degrees of asthma symptom severity. Step 1 is considered intermittent asthma and requires treatment with only a short-acting beta agonist as needed. Step 2 is considered mild persistent asthma and is treated with a low-dose inhaled corticosteroid (ICS), cromolyn, theophylline or a leukotriene receptor antagonist (LTRA). Steps 3 and 4 are considered moderate persistent asthma. Step 3 asthmatic patients require treatment with either a mediumdose ICS alone or a low-dose ICS in addition to either a long acting beta agonist (LABA), LTRA or theophylline. Step 4 asthmatic patients require medium-dose ICSs in addition to either a LABA, LTRA or theophylline. Steps 5 and 6 are considered severe persistent asthma. Step 5 asthmatic patients require high-dose ICS in addition to either a LABA, LTRA or theophylline for symptom control, whereas step 6 asthmatic patients have the same requirements as a step 5 patient, in addition to oral systemic corticosteroids. None of our study patients met the criteria for step 6 , as all study subjects who were receiving oral systemic corticosteroids were taking them for diseases other than asthma and were therefore excluded.

\section{Statistics}

Statistical analyses were performed using the SPSS Predictive Analytics SoftWare v.19 (IBM Corporation, Somers, NY) and Microsoft Excel (2007). Means and standard deviations were calculated for descriptive summary statistics and 25(OH)D measurements. Anthropometrics and 25(OH)D levels were compared using Student's t-test. Seasons were compared using Fisher's exact test. Data were expressed as mean $\pm \mathrm{SD}$.

\section{Results}

\section{Subject characteristics}

We identified 9445 asthmatic children and adolescents of ages 2-19 years in our database. Of these, 646 (6.84\%) had serum 25(OH)D levels available. The mean 25(OH)D of this cohort was $28.81 \pm 12.07 \mathrm{ng} / \mathrm{mL}$. Of the subjects, 263 [40.71\% of the asthmatic subjects with serum 25(OH)D levels, $2.78 \%$ of all asthmatic children] met the inclusion and exclusion criteria of this study. A group of 284 similarly aged non-asthmatic controls with available serum 25(OH)D data was identified in the same database.

A comparison of the characteristics of the asthmatic and non-asthmatic groups showed no significant differences in gender, height, weight, race or the season of vitamin D measurement. There were no differences in $25(\mathrm{OH}) \mathrm{D}$ levels in patients with asthma compared to the normal controls $(28.64 \pm 10.09$ vs. $28.42 \pm 11.47, \mathrm{p}=1.0)$. The asthmatic subjects were older than the controls (14.34 \pm 3.86 vs. $13.05 \pm 4.23, p=0.0002)$.

However, when subjects were stratified into obese and non-obese groups based on BMIs of <95th percentile for non-obese, or $>95$ th percentile for obese, (Table 1), there was a significant difference in $25(\mathrm{OH}) \mathrm{D}$ levels between obese and non-obese asthmatic patients (23.33 \pm 7.67 vs. $30.16 \pm 10.20, \mathrm{p}<0.0001)$ as well as obese and non-obese controls $(24.56 \pm 9.90$ vs. $29.50 \pm 11.66, p=0.003)$. In subsequent analysis, we stratified the subjects based on race into black and non-black. We found that that among the non-black asthmatic patients, the non-obese patients had significantly higher 25(OH)D levels compared to obese patients $(30.13 \pm 10.01$ vs. $22.84 \pm 7.85$, p<0.0001), but $25(\mathrm{OH})$ $\mathrm{D}$ levels did not differ significantly between the non-obese 


\begin{tabular}{|c|c|c|c|c|c|c|}
\hline & & & sthmatics & & & sthmatics \\
\hline & Obese & Non-obese & p-Value & Obese & Non-obese & p-Value \\
\hline & $(n=59)$ & $(n=204)$ & & $(n=62)$ & $(n=222)$ & \\
\hline Age, years & $14.95 \pm 3.17$ & $14.16 \pm 4.03$ & 0.12 & $14.03 \pm 3.23$ & $12.78 \pm 4.43$ & 0.014 \\
\hline Males & $\begin{array}{r}25 / 59 \\
(42.4 \%)\end{array}$ & $\begin{array}{r}96 / 204 \\
(47.0 \%)\end{array}$ & 0.53 & $\begin{array}{r}25 / 62 \\
(40.3 \%)\end{array}$ & $\begin{array}{r}94 / 230 \\
(42.3 \%)\end{array}$ & 0.92 \\
\hline Height SDS & $0.02 \pm 1.27$ & $-0.09 \pm 1.31$ & 0.58 & $0.51 \pm 1.20$ & $-0.16 \pm 1.13$ & $<0.0001$ \\
\hline Weight SDS & $2.05 \pm 0.55$ & $0.17 \pm 1.18$ & $<0.0001$ & $2.34 \pm 0.56$ & $-0.02 \pm 1.10$ & $<0.0001$ \\
\hline BMI SDS & $2.10 \pm 0.29$ & $0.32 \pm 0.85$ & $<0.0001$ & $2.23 \pm 0.36$ & $0.06 \pm 1.03$ & $<0.0001$ \\
\hline Race (non-black) & $\begin{array}{r}49 / 59 \\
(83.0 \%)\end{array}$ & $\begin{array}{r}180 / 204 \\
(88.0 \%)\end{array}$ & 0.30 & $\begin{array}{r}48 / 62 \\
(77.4 \%)\end{array}$ & $\begin{array}{r}195 / 222 \\
(87.8 \%)\end{array}$ & 0.04 \\
\hline Serum $25(\mathrm{OH}) \mathrm{D}$ & $23.33 \pm 7.67$ & $30.16 \pm 10.20$ & $<0.0001$ & $24.56 \pm 9.90$ & $29.50 \pm 11.66$ & 0.003 \\
\hline Season (Summer and Autumn) & $\begin{array}{r}37 / 59 \\
(62.7 \%)\end{array}$ & $\begin{array}{r}108 / 204 \\
(52.9 \%)\end{array}$ & 0.18 & $\begin{array}{r}44 / 62 \\
(71.0 \%)\end{array}$ & $\begin{array}{r}125 / 222 \\
(56.3 \%)\end{array}$ & 0.04 \\
\hline
\end{tabular}

Table 1 Comparison of the body mass index adjusted characteristics of asthmatic patients and controls. SDS, standard deviation score; 25(OH)D, 25-hydroxyvitamin D; BMI, body mass index.

and obese black asthmatic patients $(29.38 \pm 11.71$ vs. $24.10 \pm 8.44, p=0.2)$. Equally, among the controls, $25(\mathrm{OH})$ $\mathrm{D}$ was significantly higher in the non-obese, non-black group vs. the obese, non-black group (30.45 \pm 11.66 vs. $26.21 \pm 8.81, \mathrm{p}=0.006)$, but did not differ between the nonobese and obese black controls $(22.63 \pm 9.30$ vs. $18.92 \pm 11.62$, $\mathrm{p}=0.3)$. There was neither a difference in 25(OH)D levels between asthmatic males vs. females $(28.81 \pm 10.74$ vs. $28.19 \pm 9.80, \mathrm{p}=0.6$ ), nor between male and female controls (28.86 \pm 11.83 vs. $28.11 \pm 11.23, \mathrm{p}=0.6)$.

There was no significant difference in mean $25(\mathrm{OH})$ D levels when stratified according to the NHLBI's asthma symptom severity (1). The mean \pm standard deviations for serum $25(\mathrm{OH}) \mathrm{D}$ for the five asthma steps are as follows: $28.30 \pm 9.83$ for step $1,30.26 \pm 10.64$ for step $2,28.25 \pm 11.43$ for step 3, $26.60 \pm 8.38$ for step 4 and $25.00 \pm 6.70$ for step 5 $(\mathrm{p}=0.5)$ (Figure 1). Table 2 shows the proportional analysis of vitamin D status of the asthmatic subjects based on the five steps of asthma symptom severity.

\section{Discussion}

Our case-control study did not detect any significant difference in $25(\mathrm{OH}) \mathrm{D}$ levels in patients with asthma compared to the normal controls. Our sub-analysis showed that mean 25(OH)D levels were significantly lower in both the obese controls and asthmatic patients compared to their respective normal weight peers. We found no relationship between serum 25(OH)D concentrations and the steps of asthma severity.
Several studies have investigated the relationship between vitamin D and asthma in children (13-16) and adults (20) with conflicting results. Our finding of no significant difference in mean 25(OH)D level between asthmatic patients and controls is consistent with the findings from two case control studies by Devereux et al. (20) and Goleva et al. (17), but differed from the results of Brehm (13), Chinellato et al. (14), Alyasin et al. (15) and Gupta et al. (16). Brehm et al. (13) compared the proportions of 616 asthmatic subjects of ages 6-14 years who meet the criteria for vitamin D sufficiency ( $>30 \mathrm{ng} / \mathrm{mL}$ ), insufficiency (20-30 ng/mL) and deficiency (<20 ng/mL). They found

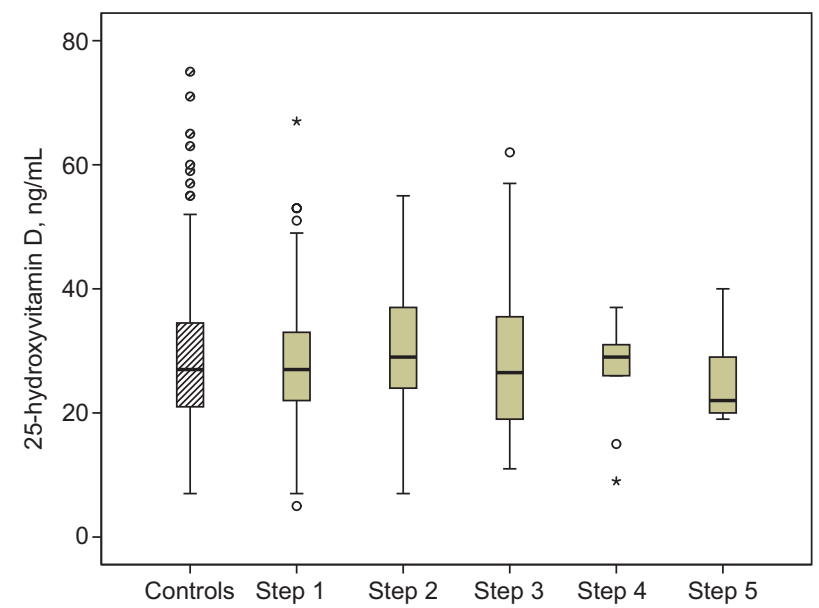

Figure 1 Box plots showing no significant differences in mean 25 -hydroxyvitamin D levels between the controls and asthmatic patients stratified according to the five steps of asthma symptom severity. 


\begin{tabular}{|c|c|c|c|c|c|}
\hline & Step 1 & Step 2 & Step 3 & Step 4 & Step 5 \\
\hline & $(n=138)$ & $(n=61)$ & $(n=44)$ & $(n=10)$ & $(n=10)$ \\
\hline Mean 25(OH)D & $28.30 \pm 9.83$ & $30.26 \pm 10.64$ & $28.25 \pm 11.43$ & $26.60 \pm 8.38$ & $25.00 \pm 6.7$ \\
\hline Vitamin D sufficient & $114 / 138(82.61 \%)$ & $50 / 61$ & $33 / 44$ & $8 / 10$ & $9 / 10$ \\
\hline$(\geq 20 \mathrm{ng} / \mathrm{mL})$ & & (81.97\%) & $(75.00 \%)$ & $(80.00 \%)$ & (90.00\%) \\
\hline Vitamin D insufficient & $15 / 138(10.87 \%)$ & $8 / 61$ & $6 / 44$ & $0 / 10$ & $1 / 10$ \\
\hline$(16-19 \mathrm{ng} / \mathrm{mL})$ & & (13.11\%) & (13.64\%) & $(0 \%)$ & $(10.00 \%)$ \\
\hline Vitamin D deficient & $9 / 138$ & $3 / 61$ & $5 / 44$ & $2 / 10$ & $0 / 10$ \\
\hline$(\leq 15 \mathrm{ng} / \mathrm{mL})$ & $(6.52 \%)$ & $(4.92 \%)$ & $(11.36 \%)$ & $(20.00 \%)$ & $(0 \%)$ \\
\hline
\end{tabular}

Table 2 Proportional Analysis of Vitamin D status across the five steps of asthma symptom severity. 25(OH)D, 25-hydroxyvitamin D

that lower serum 25(OH)D levels were associated with increased asthma severity as determined by pulmonary function testing (PFT) and frequency of asthma-related hospitalizations. This study found a significant inverse correlation between serum 25(OH)D levels and frequency of asthma exacerbations. However, unlike our study, it did not employ a case-control model to investigate the differences in mean 25(OH)D levels between asthmatic patients and controls.

In a cross-sectional study of a cohort of 75 asthmatic Italian children aged 5-11 years, Chinellato et al. (14) found that the asthmatic children with higher serum $25(\mathrm{OH})$ D levels had better perceived control of their symptoms compared to asthmatic patients with lower serum $25(\mathrm{OH})$ D levels. This study also showed that, after stratifying the asthmatic patients into controlled, partly controlled and uncontrolled groups, serum 25(OH)D levels were significantly higher in the controlled group compared to the partly controlled group. This study was similar to our study because it was designed to investigate the relationship between serum 25(OH)D levels and asthma severity. However, our stratification of asthma severity was based on controller medications and their dosages as outlined by the NHLBI's guidelines (1), whereas the study by Chinellato et al. categorized severity based on the Global Initiative for Asthma guidelines.

Gupta et al. (16) evaluated the relationship between serum 25(OH)D and asthma severity by comparing the median 25(OH)D levels of subjects with severe steroid resistant asthma, moderate asthma and non-asthmatic controls, as well as the frequency of vitamin D deficiency defined as serum 25(OH)D levels of $<20 \mathrm{ng} / \mathrm{mL} \quad(<50$ $\mathrm{nmol} / \mathrm{L})$ in the study. They reported significantly lower serum 25(OH)D levels in patients with steroid-resistant asthma compared with those with moderate asthma and non-asthmatic controls. They also found a higher prevalence of vitamin D deficiency and insufficiency in subjects with steroid resistant asthma. Alyasin et al. (15) compared the mean serum $25(\mathrm{OH}) \mathrm{D}$ levels of patients of ages $6-18$ years in Iran. They found a significant difference in mean 25(OH)D levels between 50 asthmatic patients and 50 agematched controls.

Some studies have reached opposite conclusions. Devereux et al. (20) reported in a case-control study involving 80 asthmatic patients and 80 controls in two separate centers in Europe, Aberdeen (latitude $57^{\circ} \mathrm{N}$ ) and Norwich (latitude $52^{\circ} \mathrm{N}$ ), that there was no significant difference in serum 25(OH)D concentration between asthmatic patients and controls overall, or in Aberdeen or Norwich. In a casecontrol study of both pediatric and adult patients with asthma, Goleva et al. (17) noted no significant difference in the prevalence of vitamin D deficiency/insufficiency in their asthmatic group and normal controls. Hypponen et al. reported that regular high dose ( $\geq 2000 \mathrm{IU} / \mathrm{d}$ ) vitamin D supplementation during infancy has been associated with increased likelihood of atopy and allergic rhinitis at 31 years (22), whereas Gale et al. showed that high $(>30$ $\mathrm{ng} / \mathrm{mL}$ ) maternal serum 25(OH)D levels during pregnancy were associated with an increased likelihood of childhood eczema at 9 months and asthma at 9 years (21). Thus, there is no consensus on the relationship between vitamin D and asthma.

The finding of a lack of relationship between vitamin $\mathrm{D}$ and asthma in our study is consistent with the report by Devereux et al. (20), but differed from the conclusions of other studies that showed a relationship between serum 25(OH)D concentrations and asthma symptom severity $(12,13)$. These association studies suggest that vitamin D could exert some beneficial effects in the treatment of steroid-resistant patients with asthma (3). This concept of a steroid-sparing effect of vitamin D was informed from a study that reported that the addition of vitamin $\mathrm{D}$ and dexamethasone to cultures of $\mathrm{CD}^{+} \mathrm{T}$ regulatory cells from steroid resistant asthmatic patients enhanced 
interleukin-10 secretion from these cells to levels comparable to those from cells of steroid-sensitive patients treated with dexamethasone alone (28). However, some experiments in knock out (KO) mice do not support this association. Wittke et al. (29) conducted an experimental allergic asthma induction in VDR KO mice and wild-type (WT) mice. The WT mice developed asthma as expected, but the KO did not. The administration of 1,25-dihydroxyvitamin D to the WT mice had no effect on asthma severity. The authors explained that the inability of the VDR KO mice to develop asthma was due to a failure of the lung micro-environment to attract pathogenic cells to the lungs rather than a failure of the priming and activation of immune cells. This finding is supported by a recent study that showed that the inability of VDR KO mice to develop airway hyperreactivity is a result of defective invariant natural killer cells and not due to defective Th2 cells (30). Furthermore, even though some studies have described an association between VDR genetic polymorphisms and asthma, this association has not been replicated in subsequent experiments (6). Therefore, the role of vitamin D on asthma symptom severity is inconclusive.

Our study showed that that mean 25(OH)D levels were significantly lower in both the obese controls and asthmatic patients compared to their respective normal weight peers. This is consistent with earlier reports of vitamin D deficiency in obese individuals (31-34). The etiology of vitamin D deficiency in obesity is unclear. Proposed causative factors include poor general nutrition, which results in low intake of vitamin D-containing foods such as salmon, inadequate exposure to sunlight and the sequestration of vitamin D in fat stores in obese individuals, which makes vitamin D unavailable for biological activities (32).

When compared to other studies that detected a significant difference between asthmatic groups and controls, our study of 263 asthmatic patients and 284 controls was sufficiently powered to detect any significant difference in 25(OH)D levels between the asthmatic patients and normal controls and any correlation with asthma severity. A similar case control study involving 50 asthmatic children and 50 controls found a significant difference in 25(OH)D levels and also a significant inverse correlation with asthma severity (15). However, another study (20), powered to detect an $18 \%$ difference in total 25(OH)D levels failed to detect either a significant difference in 25(OH)D levels or a correlation with asthma severity in a similar case-control study in 80 asthmatic adults and 80 controls. A third case-control study involving children and adults reported a non-significant difference in 25(OH)D levels between 103 asthmatic patients and 102 controls. This study, however, showed a significant inverse association between vitamin $\mathrm{D}$ and serum IgE, as well as vitamin $\mathrm{D}$ and daily inhaled corticosteroid dose in the pediatric asthma group only (17). Among the non casecontrol studies that reported significant inverse correlation with asthma severity, one study evaluated 616 asthmatic patients (13), another studied 75 asthmatic patients (14), whereas a third study investigated 86 asthmatic patients (16). Therefore, more carefully designed studies are needed to clarify the relationship between asthma severity and vitamin D status.

There are a number of limitations to be considered in the interpretation of our results. The first shortcoming is the cross-sectional design of the study which makes it difficult to establish causality. We also had a small sample size for the asthma steps 4 and 5 . Such a sample size would be inadequate to detect subtle differences between the groups and could have resulted in type 2 error. In contrast, we had a representative number of patients in asthma steps 1, 2, and 3 to accurately assess the relationship between vitamin $\mathrm{D}$ and asthma symptom severity in these steps. Another factor to take into consideration in interpreting our results is the accuracy of documentation of patients' disease state. In addition to documented asthma exacerbations and prescriptions filled for these exacerbations, we relied on physician's documentation of the patients' medications as a means of evaluating asthma symptom severity. There was neither a way to confirm patients' compliance with the listed medications, nor was there a way to know if there had been recent changes to patients' medications based on changes in symptom severity. Some of the strengths of this study include the fact that the case-control design enabled us to evaluate a large cohort of patients with asthma and compared their data to a control group. We also used the NHLBI criteria for the delineation of asthma symptom severity.

In conclusion, we did not detect any significant differences in 25(OH)D levels between asthmatic patients and their age-matched controls. We noted that mean $25(\mathrm{OH})$ D levels were significantly lower in both the obese controls and asthmatic patients compared to their respective normal weight peers. We found no relationship between serum 25(OH)D concentrations and the NHLBI's steps of asthma severity. Our study lends support to the Institute of Medicine's recent position that there was no conclusive evidence for vitamin D supplementation in asthma (24). Randomized controlled trials of vitamin D supplementation in asthma are warranted to determine the role of vitamin D on asthma symptom severity.

Received June 1, 2012; accepted June 9, 2012;

previously published online July 27, 2012 


\section{References}

1. Expert Panel Report 3 (EPR-3): Guidelines for the diagnosis and management of asthma-Summary Report 2007. J Allergy Clin Immunol 2007;120:S94-138.

2. Akinbami LJ, Moorman JE, Liu X. Asthma prevalence, health care use, and mortality: United States, 2005-2009. Natl Health Stat Report 2011;12:1-14.

3. Banerjee A, Damera G, Bhandare R, Gu S, Lopez-Boado Y, et al. Vitamin $D$ and glucocorticoids differentially modulate chemokine expression in human airway smooth muscle cells. Br J Pharmacol 2008;155:84-92.

4. Paul G, Brehm JM, Alcorn JF, Holguin F, Aujla SJ, et al. Vitamin D and asthma. Am J Respir Crit Care Med 2012;185:124-32.

5. Krstic G. Asthma prevalence associated with geographical latitude and regional insolation in the United States of America and Australia. PloS One 2011;6:e18492.

6. Litonjua AA, Weiss ST. Is vitamin D deficiency to blame for the asthma epidemic? J Allergy Clin Immunol 2007;120:1031-5.

7. Provvedini DM, Tsoukas CD, Deftos LJ, Manolagas SC. 1,25-dihydroxyvitamin D3 receptors in human leukocytes. Science 1983;221:1181-3.

8. Bhalla AK, Amento EP, Clemens TL, Holick MF, Krane SM. Specific high-affinity receptors for 1,25-dihydroxyvitamin D3 in human peripheral blood mononuclear cells: presence in monocytes and induction in T lymphocytes following activation. J Clin Endocrinol Metab 1983;57:1308-10.

9. Hansdottir S, Monick MM, Hinde SL, Lovan N, Look DC, et al. Respiratory epithelial cells convert inactive vitamin $D$ to its active form: potential effects on host defense. J Immunol 2008;181:7090-9.

10. Bosse Y, Maghni K, Hudson TJ. 1alpha,25-dihydroxy-vitamin D3 stimulation of bronchial smooth muscle cells induces autocrine, contractility, and remodeling processes. Physiol Genomics 2007;29:161-8.

11. Iqbal SF, Freishtat RJ. Mechanism of action of vitamin D in the asthmatic lung. J Investig Med 2011;59:1200-2.

12. Searing DA, Zhang Y, Murphy JR, Hauk PJ, Goleva E, et al. Decreased serum vitamin $D$ levels in children with asthma are associated with increased corticosteroid use. J Allergy Clin Immunol 2010;125:995-1000.

13. Brehm JM, Celedon JC, Soto-Quiros ME, Avila L, Hunninghake GM, et al. Serum vitamin D levels and markers of severity of childhood asthma in Costa Rica. Am J Respir Crit Care Med 2009;179:765-71.

14. Chinellato I, Piazza M, Sandri M, Peroni D, Piacentini G, et al. Vitamin D serum levels and markers of asthma control in Italian children. J Pediatr 2011;158:437-41.

15. Alyasin S, Momen T, Kashef S, Alipour A, Amin R. The relationship between serum 25 hydroxy vitamin $D$ levels and asthma in children. Allergy Asthma Immunol Res 2011;3:251-5.

16. Gupta A, Sjoukes A, Richards D, Banva W, Hawrylowicz C, et al. Relationship between serum vitamin $D$, disease severity, and airway remodeling in children with asthma. Am J Respir Crit Care Med 2011;184:1342-9.

17. Goleva E, Searing DA, Jackson LP, Richers BN, Leung DY. Steroid requirements and immune associations with vitamin $D$ are stronger in children than adults with asthma. J Allergy Clin Immunol 2012;129:1243-51.
18. Morales E, Romieu I, Guerra S, Ballester F, Rebagliato M, et al. Maternal vitamin D status in pregnancy and risk of lower respiratory tract infections, wheezing, and asthma in offspring. Epidemiology 2012;23:64-71.

19. Camargo CA Jr, Rifas-Shiman SL, Litonjua AA, Rich-Edwards JW, Weiss ST, et al. Maternal intake of vitamin D during pregnancy and risk of recurrent wheeze in children at $3 \mathrm{y}$ of age. Am J Clin Nutr 2007;85:788-95.

20. Devereux G, Wilson A, Avenell A, McNeill G, Fraser WD. A case-control study of vitamin D status and asthma in adults. Allergy 2010;65:666-7.

21. Gale CR, Robinson SM, Harvey NC, Javaid MK, Jiang B, et al. Maternal vitamin $D$ status during pregnancy and child outcomes. Eur J Clin Nutr 2008; 62:68-77.

22. Hypponen E, Sovio U, Wjst M, Patel S, Pekkanen J, et al. Infant vitamin d supplementation and allergic conditions in adulthood: northern Finland birth cohort 1966. Ann N Y Acad Sci 2004;1037:84-95.

23. Wjst M, Hypponen E. Vitamin D serum levels and allergic rhinitis. Allergy 2007;62:1085-6.

24. Ross AC, Manson JE, Abrams SA, Aloia JF, Brannon PM, et al. The 2011 report on dietary reference intakes for calcium and vitamin $\mathrm{d}$ from the institute of medicine: what clinicians need to know. J Clin Endocrinol Metab 2011;96:53-8.

25. Barlow SE. Expert committee recommendations regarding the prevention, assessment, and treatment of child and adolescent overweight and obesity: summary report. Pediatrics 2007;120(Suppl 4):S164-92.

26. Kuczmarski RJ, Ogden CL, Guo SS, Grummer-Strawn LM, Flegal KM, et al. 2000 CDC growth charts for the United States: methods and development. Vital Health Stat 2002;11: 1-190.

27. Misra M, Pacaud D, Petryk A, Collett-Solberg PF, Kappy M. Vitamin $D$ deficiency in children and its management: review of current knowledge and recommendations. Pediatrics 2008;122:398-417.

28. Xystrakis E, Kusumakar S, Boswell S, Peek E, Urry Z, et al. Reversing the defective induction of IL-10-secreting regulatory T cells in glucocorticoid-resistant asthma patients. J Clin Invest 2006;116:146-55.

29. Wittke A, Weaver V, Mahon BD, August A, Cantorna MT. Vitamin $D$ receptor-deficient mice fail to develop experimental allergic asthma. J Immunol 2004;173:3432-6.

30. Yu S, Zhao J, Cantorna MT. Invariant NKT cell defects in vitamin $D$ receptor knockout mice prevents experimental lung inflammation. J Immunol 2011;187:4907-12.

31. Parikh SJ, Edelman M, Uwaifo GI, Freedman RJ, SemegaJanneh $M$, et al. The relationship between obesity and serum 1,25-dihydroxy vitamin $\mathrm{D}$ concentrations in healthy adults. J Clin Endocrinol Metab 2004;89:1196-9.

32. Liel Y, Ulmer E, Shary J, Hollis BW, Bell NH. Low circulating vitamin D in obesity. Calcif Tissue Int 1988;43:199-201.

33. Bell NH, Epstein S, Greene A, Shary J, Oexmann MJ, et al. Evidence for alteration of the vitamin D-endocrine system in obese subjects. J Clin Invest 1985;76:370-3.

34. Zamboni G, Soffiati M, Giavarina D, Tato L. Mineral metabolism in obese children. Acta Paediatr Scand 1988;77:741-6. 